\section{NEW CAUSES OF OLD DISEASES. ARE WE READY?}

Ken L. Wan $^{1}$, Thushari K. Vithanage ${ }^{1}$, Zhong X. Lu ${ }^{1,2}$, James C. G. Doery ${ }^{1,2}$

${ }^{1}$ Monash Pathology, Monash Health, Clayton, VIC; and ${ }^{2}$ Department of Medicine, Monash University, Clayton, VIC, Australia

A 67-year-old male with metastatic cholangiocarcinoma presented with dehydration and delirium approximately 6 weeks after participating into a Rare Cancer Trial utilising combination of immune checkpoint inhibitors (ICIs), i.e., ipilimumab and nivolumab.

His electrolytes were normal but cortisol was $36 \mathrm{nmol} / \mathrm{L}$ (185$625)$ and ACTH was $2 \mathrm{pmol} / \mathrm{L}(<10)$. Sixty-minute postSynacthen cortisol was $153 \mathrm{nmol} / \mathrm{L}$ (>530). Secondary hypoadrenalism was diagnosed on the basis of low ACTH and baseline cortisol with poor response to Synacthen. Thyroid function tests revealed thyrotoxicosis, while TPO, thyroglobulin and TSH receptor antibodies were all negative.

ICIs have been increasingly implicated in autoimmune endocrinopathies. Combination of ipilimumab and nivolumab can give rise to hypopituitarism or hypophysitis, and thyroid dysfunction. In healthy individuals, immune checkpoints maintain immunological tolerance to self-antigens. By inhibiting these immune checkpoints, ICIs cause auto-immune like manifestations against multiple organs. Typically, complications develop in susceptible patients 6-15 weeks after introduction of ICIs. The preferred screening test for hypophysitis is morning cortisol and ACTH, followed by Synacthen test. ${ }^{1}$

In oncology, ICIs are the most rapidly expanding class of drugs alternatives to traditional chemotherapy. Therefore, oncologists, endocrinologists and pathologists need to understand their mechanism of action, side effects, importance of monitoring ICI usage to detect and investigate the associated endocrine disorders.

\section{Reference}

1. Barroso-Sousa R, Ott PA, Hodi FS, et al. Endocrine dysfunction induced by immune checkpoint inhibitors: practical recommendations for diagnosis and clinical management. Cancer 2018; 124: 1111-21.

\section{PARATHYROID HORMONE (PTH) IN HYPERCALCAEMIC PATIENTS. ANALYTICAL COMPARISON OF EIGHT PTH IMMUNOASSAYS}

J. Douglas, J. Hepburn, K. Young, J. Nicolson, J. Smith, B. Teis, R. Flatman, G. Ward, D. Kanowski, L. Price Sullivan Nicolaides Pathology, Biochemistry Dept, Bowen Hills, Qld, Australia

Introduction: Modern PTH immunoassays can be classified as second (intact PTH) or third generation assays (PTH 1-84). It is generally considered that the performance in primary hyperparathyroidism (PHPT) of second and third generation assays is similar. The aim of this study was to compare PTH immunoassays in hypercalcaemic patients.

Methods: EDTA plasma from 112 hypercalcaemic patients with eGFR $>60$ was frozen prior to immunoassay. PTH assays were as follows: Roche (intact); Roche (PTH 1-84); Abbott Architect; DiaSorin N-Tact; DiaSorin 1-84 PTH; Beckman Access; Siemens Centaur; Siemens Immulite 2000; and SNIBE Maglumi 2000.
Results: Eighty-five patients had PTH levels $>$ upper limit of the RI and 91/112 patients had PHPT.

Passing-Bablok analysis versus Roche Intact PTH slopes were as follows: 0.45 (DiaSorin 1-84 PTH); 0.89 (Beckman Access); 0.96 (Siemens Centaur); 1.08 (Abbott Architect); 1.28 (SNIBE Maglumi 2000); 1.28 (DiaSorin N-Tact); and 1.39 (Siemens Immulite 2000). Similar slopes were observed in normocalcaemic reference patients.

Conclusions: This study confirms observations that Intact PTH and PTH 1-84 assays have similar diagnostic utility in PHPT. Better standardisation of PTH assays would improve comparability of PTH assays in patients without renal disease. Standardisation of 3rd generation PTH 1-84 assays would result in comparable PTH levels regardless of eGFR.

\section{'SILVER MAN' - A FIRST CASE REPORT OF SILVER ASSOCIATED STEATOHEPATITIS (SASH) AND DISCUSSION OF ITS PATHOMECHANISM}

Ka Chung Wong ${ }^{1}$, Tsz Ki Ling ${ }^{1}$, Florence Loong ${ }^{2}$, Patrick Siu-chung Leung ${ }^{3}$, Chun Yiu Law ${ }^{1}$, Ching Wan Lam ${ }^{1,4}$ ${ }^{1}$ Division of Chemical Pathology, Department of Pathology, Queen Mary Hospital, Hong Kong, China; ${ }^{2}$ Division of Anatomical Pathology, Department of Pathology, Queen Mary Hospital, Hong Kong, China; ${ }^{3}$ Department of Accident and Emergency Department, Queen Mary Hospital, Hong Kong, China; and ${ }^{4}$ Department of Pathology, University of Hong Kong, Hong Kong, China

A 47-year-old Chinese man, who worked as a clerk in the electronics industry, presented with gait instability and gloveand-stocking sensation. He had anaemia, deranged liver function, low ceruloplasmin $(0.05 \mathrm{~g} / \mathrm{L}$; ref $0.18-0.35)$, high 24 -urine copper $(2.02 \mu \mathrm{mol} / \mathrm{L}$; ref $<0.5 \mu \mathrm{mol} / \mathrm{L})$, low iron level $(5 \mathrm{umol} / \mathrm{L}$; ref 9-33), high ferritin (4521 pmol/L; ref 52-738) and low transferrin saturation (11\%; ref 16-45). MRI liver and pancreas showed homogenous T2 hypointensity, a feature of iron deposition. MRI brain showed artefacts in bilateral globus pallidi that suggested mineralisation. Sequencing of $A T P 7 B$ gene did not reveal any pathogenic variants. Further clarification of the clinical history revealed that colloidal silver was prescribed by his private practitioner for over a year. His blood silver level was about a thousand times above that of controls. In the liver tissues, there were patchy lymphocytic infiltrates with periportal and focal bridging fibrosis, moderate haemosiderin deposits and presence of silver. Clinical symptoms and biochemical results were improved after silver exposure was stopped. Silver binds with ceruloplasmin and loses its ferroxidase activity, so $\mathrm{Fe} 2+$ cannot be oxidised to $\mathrm{Fe} 3+$ and cannot be carried by transferrin. This leads to intracellular iron overload in the liver, pancreas and neuronal tissues.

\section{SEASONAL EFFECTS ON DEATH FROM DIABETIC KETOACIDOSIS}

$\underline{\text { Sunisha Chahal }}^{1}$, Suneeth Mathew ${ }^{1}$, Nicole Loper $^{2}$, Kilak Kesha $^{1}$, Simon Stables ${ }^{1}$, Paul Morrow ${ }^{1}$, Charley Glenn ${ }^{1}$, Rexson Tse

${ }^{I}$ Department of Forensic Pathology, LabPLUS, Auckland City Hospital, Auckland, New Zealand; and ${ }^{2}$ Faculty of Medical and 